\title{
ADNP syndrome
}

INSERM

\section{Source}

INSERM. (1999). Orphanet: an online rare disease and orphan drug data base. ADNP syndrome. ORPHA:404448

A rare syndromic intellectual disability characterized by global developmental delay, gastrointestinal problems, hypotonia, delayed speech, behavioral and sleep problems, pain insensitivity, seizures, structural brain anomalies, dysmorphic features, visual problems, early tooth eruption and autistic features. 